MATHEMATICS OF COMPUTATION

Volume 74, Number 249, Pages 279-290

S 0025-5718(04)01648-5

Article electronically published on April 16, 2004

\title{
RECOVERING SIGNALS FROM INNER PRODUCTS INVOLVING PROLATE SPHEROIDALS IN THE PRESENCE OF JITTER
}

\author{
DOROTA DABBROWSKA
}

\begin{abstract}
The paper deals with recovering band- and energy-limited signals from a finite set of perturbed inner products involving the prolate spheroidal wavefunctions. The measurement noise (bounded by $\delta$ ) and jitter meant as perturbation of the ends of the integration interval (bounded by $\gamma$ ) are considered. The upper and lower bounds on the radius of information are established. We show how the error of the best algorithms depends on $\gamma$ and $\delta$. We prove that jitter causes error of order $\Omega^{\frac{3}{2}} \gamma$, where $[-\Omega, \Omega]$ is a bandwidth, which is similar to the error caused by jitter in the case of recovering signals from samples.
\end{abstract}

\section{INTRODUCTION AND PRELIMINARIES}

The usual approach to recovery of signals [1, 4], [5], 6], 7], [8], [9, [10] assumes gathering their samples, which are then combined in some way to get approximate signals' values. In practice, the data is contaminated with some noise coming from an inaccurate sample points reading (called jitter) and from the measurements of resulting samples. It has been proved [1] that to get satisfactory quality of the reconstruction, we may need to ensure very small jitter.

In the 1960's, mainly due to Slepian, Landau and Pollak [14, 20], 22], a new way of gathering information about signals came into consideration. Instead of samples, certain inner products involving prolate spheroidal wavefunctions could be evaluated. Optimality properties of such information have been exhibited in 8], 10]. The measurement noise has been proved to have smaller influence on the quality of recovery than in the case of utilising information consisting of signals' samples 4 .

We deal with the recovery of band- and energy-limited signals. We estimate the loss caused by jitter (meant here as the perturbation of the length of the integration interval) while using the prolate spheroidal wavefunctions.

Received by the editor July 19, 2002 and, in revised form, June 2, 2003.

2000 Mathematics Subject Classification. Primary 68Q17, 94A12, 94A11; Secondary 94A20, $65 \mathrm{G} 99$

Key words and phrases. Problem complexity, signal theory, application of orthogonal functions in communication.

(C)2004 American Mathematical Society 
In order to give a precise formulation of the problem, we denote by $F$ the space of signals of bandwidth $[-\Omega, \Omega]$,

$$
F=\left\{f: \mathbb{R} \rightarrow \mathbb{C}: f(t)=\int_{-\Omega}^{\Omega} \hat{f}(\omega) e^{\mathrm{it} \omega} \mathrm{d} \omega, \hat{f} \in L^{2}[-\Omega, \Omega]\right\} .
$$

We are interested in the signals with limited energy, that is, in the subclass $E=$ $\{f \in F: \hat{f} \in J\}$, where $J$ denotes the unit ball in $L^{2}[-\Omega, \Omega]$. The reconstruction of a signal $f$ at a point $t_{0}$ from the interval $[-\tau, \tau], \tau>0$, can be treated as a recovery of the linear functional

$$
\begin{aligned}
& S: F \rightarrow \mathbb{C}, \\
& S(f)=f\left(t_{0}\right),
\end{aligned}
$$

from information vector $\vec{y} \in \mathbb{N}(f)$, where $\mathbb{N}: E \rightarrow 2^{\mathbb{C}^{n}}$ is an information operator defined by

$$
\begin{array}{r}
\mathbb{N}(f)=\left\{\vec{y} \in \mathbb{C}^{n}: \quad y_{k}=\int_{-\tau+\Lambda_{k}}^{\tau+\Gamma_{k}} f(t) w_{k}(t) \mathrm{d} t+\Delta_{k},\right. \\
\left.\|\vec{\Gamma}\|_{p_{1}},\|\vec{\Lambda}\|_{p_{1}} \leq \gamma,\|\vec{\Delta}\|_{p_{2}} \leq \delta\right\} .
\end{array}
$$

Here, $\gamma$ and $\delta$ are fixed positive numbers, $1 \leq p_{1}, p_{2} \leq \infty$, and $\|\cdot\|_{p}$ denotes the $p$ th norm in $\mathbb{C}^{n}$. The functions $w_{k}$ are scaled prolate spheroidal wavefunctions as precisely defined in Section 3 We shall also use the symbols $q_{1}$ and $q_{2}$ to denote the quantities associated to $p_{1}$ and $p_{2}$, i.e.,

$$
\frac{1}{p_{1}}+\frac{1}{q_{1}}=1, \quad \frac{1}{p_{2}}+\frac{1}{q_{2}}=1,
$$

with the convention that $\frac{1}{\infty}=0$.

To recover $S(f)$, we use an algorithm $\phi: \mathbb{C}^{n} \rightarrow \mathbb{C}$, which can be an arbitrary mapping. We measure the error of $\phi$ by its worst performance in the class $E$ :

$$
e(\phi, \gamma, \delta)=\sup _{f \in E} \sup _{\vec{y} \in \mathbb{N}(f)}\|S(f)-\phi(\vec{y})\| .
$$

Our aim is to find the best possible algorithm. If some $\phi^{*}$ satisfies

$$
e\left(\phi^{*}, \gamma, \delta\right)=\inf _{\phi} e(\phi, \gamma, \delta),
$$

we call it an optimal algorithm.

Let $A(\vec{y})$ denote the image under $S$ of the set of all elements from $E$ sharing the information $\vec{y}$ :

$$
A(\vec{y})=\{S(f): f \in E, \vec{y} \in \mathbb{N}(f)\} .
$$

The quantities

$$
\begin{aligned}
r(\gamma, \delta) & =\sup _{\vec{y} \in \mathbb{C}^{n}} \inf _{g \in G} \sup _{a \in A(\vec{y})}\|g-a\|, \\
d(\gamma, \delta) & =\sup _{\vec{y} \in \mathbb{C}^{n}} \sup _{a, b \in A(\vec{y})}\|a-b\|,
\end{aligned}
$$


where the supremum over the empty set is to mean zero, define a radius and a diameter of information, respectively. It is well known that

$$
r(\gamma, \delta)=\inf _{\phi} e(\phi, \gamma, \delta)
$$

see for instance [18, [19] for the proof. We shall use these relations to estimate the lower and upper bounds on the error of the optimal algorithm. For more information on the worst case setting we refer the reader to [19], 23], 24], 25].

We shall prove that for sufficiently small $\gamma$

$$
\frac{r(0,0)+A_{1} \Omega^{\frac{3}{2}} \gamma+B \delta}{2}+O\left(\gamma^{3}+\delta^{2}\right) \leq r(\gamma, \delta) \leq r(0,0)+A_{2} \Omega^{\frac{3}{2}} \gamma+B \delta .
$$

The constants $A_{1}$ and $A_{2}$ are independent of $\Omega, \gamma$ and $\delta$. The same refers to $B$ when $w_{k}$ are suitably chosen. Jitter causes error of order $\Omega^{\frac{3}{2}} \gamma$, which is similar to the error caused by jitter in the case of recovering signals from samples. In both cases the condition $\Omega^{\frac{3}{2}} \gamma<1$ should be satisfied to get the radius of information small enough. It may force $\gamma$ to be very small, since $\Omega$ may even exceed $2^{16}$.

\section{Prolate spheroidal Wavefunctions}

This section presents some facts concerning the prolate spheroidal wavefunctions. Given positive number $c$, we consider the differential equation

$$
\left(1-t^{2}\right) u^{\prime \prime}(t)-2 t u^{\prime}(t)+\left(\kappa-c^{2} t^{2}\right) u(t)=0 .
$$

It is well known [2 that the values of the parameter $\kappa$ such that the equation has a nonzero solution can be ordered

$$
0<\kappa_{0}(c)<\kappa_{1}(c)<\cdots,
$$

and when $\kappa=\kappa_{k}(c)$, there exists exactly one solution

$$
v_{k}:[-1,1] \rightarrow \mathbb{R}
$$

such that $\int_{-1}^{1}\left|v_{k}(t)\right|^{2} \mathrm{~d} t=1$ and $v_{k}(1) \geq 0$. Let us set $c=\Omega \tau$ and define the prolate spheroidal wavefunctions by

$$
\phi_{k}(t)=\frac{1}{\sqrt{\tau}} v_{k}\left(\frac{t}{\tau}\right), k=0,1, \ldots, t \in[\tau, \tau] .
$$

\section{Theorem 1.}

(1) The functions $\phi_{k}$ continuously depend on $\Omega$ and $\tau$. For fixed $c=\Omega \tau$ they can be extended to entire functions.

(2) Each function $\phi_{k}$ is an energy-limited signal of bandwidth $[-\Omega, \Omega]$.

(3) The functions $\phi_{k}$ are even for even $k$ and odd for odd $k$.

(4) The following eigenrelations are satisfied:

$$
\begin{aligned}
& \int_{-\tau}^{\tau} \frac{\Omega}{\pi} \operatorname{Sinc}(\Omega(t-s)) \phi_{k}(s) \mathrm{d} s=\lambda_{k} \phi_{k}(t), \\
& \int_{-\tau}^{\tau} e^{\mathrm{i} \Omega \frac{t}{\tau} s} \phi_{k}(s) \mathrm{d} s=\alpha_{k} \phi_{k}(t),
\end{aligned}
$$

where $t \in \mathbb{C}, \lambda_{k} \searrow 0$ when $k \rightarrow \infty$, and $\left|\alpha_{k}\right|^{2}=\frac{2 \pi \tau}{\Omega} \lambda_{k}$. 
(5) The functions $\phi_{k}$ are orthonormal in the space $L^{2}[-\tau, \tau]$ and orthogonal in $L^{2}[-\infty, \infty]$, and $\int_{-\infty}^{\infty}\left|\phi_{k}(t)\right|^{2} \mathrm{~d} t=\frac{1}{\lambda_{k}}$.

Here and in what follows $\operatorname{Sinc}(x)$ is defined by

$$
\operatorname{Sinc}(x)=\frac{\sin (x)}{x}, x \neq 0 ; \operatorname{Sinc}(0)=1 .
$$

It should be noted that $v_{k}, \lambda_{k}, \alpha_{k}, \kappa_{k}$ depend on $c$ and $\phi_{k}$ depends on $\Omega$ and $\tau$. To simplify the notation, we do not point it out. The above theorem was proved in 8], [22]; see also [2], [3], 9], [11, [12], [13], 14], [15], 16], [17, 20], [21] for a more complete treatment.

The following lemma will be used in the next section to get an upper bound on the radius of information.

Lemma 1. For each $k=0,1, \ldots$

$$
\phi_{k}(\tau)=\sqrt{\frac{c}{2 \tau} \frac{\mathrm{d} \ln \lambda_{k}}{\mathrm{~d} c}}=\sqrt{\frac{\Omega}{2} \frac{\mathrm{d} \ln \lambda_{k}}{\mathrm{~d} c}} .
$$

Proof. According to [3], 20] we have

$$
\frac{2}{c} v_{k}^{2}(1)=\frac{\mathrm{d} \ln \lambda_{k}}{\mathrm{~d} c} .
$$

This combined with $v_{k}(1)=\sqrt{\tau} \phi_{k}(\tau)>0$ and $c=\Omega \tau$ gives the desired formula.

The next two lemmas provide tools for establishing the upper bounds on the derivatives of $\phi_{k}$ at $\tau$. We shall use them in the next section.

Lemma 2. Let us fix $c$ and $\kappa_{k}$ and set $a=\frac{\kappa_{k}-c^{2}}{2}, b=\frac{c^{2}}{2}$ and $x_{j}=\frac{v_{k}^{(j)}(1)}{v_{k}(1)}$. Then

$$
\begin{aligned}
x_{0}= & 1, \\
x_{1}= & a, \\
x_{2}= & \frac{a^{2}}{2}-\frac{a}{2}-b, \\
x_{j}= & \left(\frac{a}{j}-\frac{j-1}{2}\right) x_{j-1}-2 b \frac{j-1}{j} x_{j-2}-b \frac{(j-1)(j-2)}{j} x_{j-3}, \\
& \quad \text { for } j=3,4, \ldots
\end{aligned}
$$

Proof. The function $v_{k}$ is a solution of the differential equation

$$
\left(1-t^{2}\right) u^{\prime \prime}(t)-2 t u^{\prime}(t)+\left(\kappa-c^{2} t^{2}\right) u(t)=0
$$

with $\kappa=\kappa_{k}$. Therefore

$$
\left(1-t^{2}\right) v_{k}^{(2)}(t)-2 t v_{k}^{(1)}(t)+\left(\kappa_{k}-c^{2} t^{2}\right) v_{k}(t)=0 .
$$

This yields

$$
\left(1-t^{2}\right) v_{k}^{(3)}(t)-4 t v_{k}^{(2)}(t)+\left(\kappa_{k}-c^{2} t^{2}-2\right) v_{k}^{(1)}(t)=0 .
$$

We shall prove by induction that the following equation holds for $j=3,4, \ldots$ :

$$
\begin{aligned}
& \left(1-t^{2}\right) v_{k}^{(j+1)}(t)-2 j t v_{k}^{(j)}(t)+\left(\kappa_{k}-c^{2} t^{2}-j(j-1)\right) v_{k}^{(j-1)}(t) \\
& -2(j-1) c^{2} t v_{k}^{(j-2)}(t)-(j-1)(j-2) c^{2} v_{k}^{(j-3)}(t)=0 .
\end{aligned}
$$

It can be verified for $j=3$ by differentiating the equation (21). Let us assume that (3) is valid for some $j$. Then by differentiation we get it for $j+1$. By the definition of $a, b$ and $x_{j}$, the lemma follows by setting $t=1$ in (1), (2) and (3). 
Lemma 3. Let $a, b$ and $x_{j}$ be defined as in Lemma 2. If

$$
\beta=\max \{|a|,|b|\}>2
$$

then

$$
\left|x_{j}\right| \leq(j-1) ! \beta^{j} \text { for } j=1,2, \ldots
$$

Proof. We proceed by induction. For $j=1$ the inequality holds. As $x_{2}=\frac{a^{2}}{2}-\frac{a}{2}-b$ and

$$
\begin{aligned}
& \frac{a^{2}}{2}-\frac{a}{2}-b \leq \frac{a^{2}}{2}-\frac{a}{2} \leq \frac{\beta^{2}+\beta}{2} \leq \beta^{2}, \\
& \frac{a^{2}}{2}-\frac{a}{2}-b \geq-\frac{a}{2}-b \geq-\frac{\beta}{2}-\beta \geq-\beta^{2},
\end{aligned}
$$

we have $\left|x_{2}\right| \leq \beta^{2}$. Since $x_{3}=\frac{1}{6} a^{3}-\frac{2}{3} a^{2}+\frac{1}{2} a-\frac{5}{3} a b+b$, it follows that

$$
\left|x_{3}\right| \leq \frac{1}{6} \beta^{3}+\frac{2}{3} \beta^{2}+\frac{1}{2} \beta+\frac{5}{3} \beta^{2}+\beta<2 \beta^{3} .
$$

We now know that the lemma holds for $j=1,2,3$. For any $j \geq 4$ we assume that $\left|x_{i}\right| \leq(i-1)$ ! $\beta^{i}$ holds for all $i<j$. Then from Lemma 2 we get

$$
\begin{aligned}
\left|x_{j}\right| \leq & \left(\frac{\beta}{j}+\frac{j-1}{2}\right)(j-2) ! \beta^{j-1}+2 \beta \frac{j-1}{j}(j-3) ! \beta^{j-2} \\
& +\beta \frac{(j-1)(j-2)}{j}(j-4) ! \beta^{j-3} \\
= & (j-1) ! \beta^{j-2}\left[\frac{\beta^{2}}{j(j-1)}+\beta\left(\frac{1}{2}+\frac{2}{j(j-2)}\right)+\frac{1}{j(j-3)}\right] \\
\leq & (j-1) ! \beta^{j-2}\left(\frac{\beta^{2}}{12}+\frac{3}{4} \beta+\frac{1}{4}\right) \\
\leq & (j-1) ! \beta^{j} .
\end{aligned}
$$

The proof is complete.

Corollary 1. Let $\beta_{k}=\max \left\{\left|\frac{\kappa_{k}}{c}-c\right|, c\right\}$, where $c \geq 2$. Then

$$
\left|\phi_{k}^{(j)}(\tau)\right| \leq(j-1) !\left(\frac{\Omega \beta_{k}}{2}\right)^{j} \phi_{k}(\tau) \text { for } j=1,2, \ldots
$$

Proof. Since

$$
\phi_{k}^{(j)}(\tau)=\frac{1}{\sqrt{\tau}} \frac{1}{\tau^{j}} v_{k}^{(j)}(1)
$$

by Lemma 3 we get

$$
\begin{aligned}
\left|\frac{\phi_{k}^{(j)}(\tau)}{\phi_{k}(\tau)}\right| & =\frac{\left|\phi_{k}^{(j)}(\tau)\right|}{\phi_{k}(\tau)} \leq \frac{1}{\tau^{j}}(j-1) !\left(\max \left\{\left|\frac{\kappa_{k}-c^{2}}{2}\right|, \frac{c^{2}}{2}\right\}\right)^{j} \\
& =(j-1) !\left(\frac{\Omega \beta_{k}}{2}\right)^{j} \cdot \square
\end{aligned}
$$




\section{Estimation OF THE RADIUS OF INFORMATION}

We are now in a position to define the functions $w_{k}$. As was mentioned before, they are scaled prolate spheroidal wavefunctions, i.e,

$$
w_{k}=W_{k-1} \phi_{k-1}, k=1, \ldots, n,
$$

where the quantities $W_{k-1}$ are arbitrary positive numbers.

We remind the reader that an optimal algorithm using the exact information $(\gamma=\delta=0)[18]$ is given by

$$
\phi^{*}(\vec{y})=\sum_{k=1}^{n} y_{k} \frac{\phi_{k-1}\left(t_{0}\right)}{W_{k-1}}
$$

We shall now estimate the error of $\phi^{*}$ for arbitrary $\gamma$ and $\delta$.

Lemma 4. Let $\phi^{*}$ be defined as above. Then

$$
e\left(\phi^{*}, \gamma, \delta\right) \leq r(0,0)+\sup _{\substack{f \in E \\\left\|\Gamma n_{1} \leq \gamma\\\right\| \vec{\Lambda}\|\|_{p_{1}} \leq \gamma}} \sum_{k=0}^{n-1} A_{k}\left(f, \Gamma_{k+1}, \Lambda_{k+1}\right)\left|\phi_{k}\left(t_{0}\right)\right|+B \delta,
$$

where

$$
\begin{aligned}
& A_{k}\left(f, \Gamma_{k+1}, \Lambda_{k+1}\right)=\int_{\substack{\left[-\tau-\mid \Lambda \\
\cup\left[\tau, \tau+\left|\Gamma_{k+1}\right|,-\tau\right]\right.}}\left|f(t) \phi_{k}(t)\right| \mathrm{d} t, \\
& B=\left\|\left[\frac{\phi_{0}\left(t_{0}\right)}{W_{0}}, \ldots, \frac{\phi_{n-1}\left(t_{0}\right)}{W_{n-1}}\right]^{T}\right\|_{q_{2}} .
\end{aligned}
$$

Proof. We have

$$
\begin{aligned}
e\left(\phi^{*}, \gamma, \delta\right) \leq & \sup _{\vec{y} \in \mathbb{C}^{n}} \sup _{\substack{f \in E \\
\vec{y} \in \mathbb{N}(f)}}\left|S(f)-\phi^{*}(\vec{y})\right| \\
\leq & \sup _{f \in E}\left|S(f)-\sum_{k=1}^{n}\left(\int_{-\tau}^{\tau}\left|f(t) w_{k}(t)\right| \mathrm{d} t\right) \frac{\phi_{k-1}\left(t_{0}\right)}{W_{k-1}}\right| \\
& +\sup _{\substack{f \in E \\
\|\vec{\Gamma}\| p_{1} \leq \gamma \\
\|\vec{\Lambda}\| p_{1} \leq \gamma}} \sum_{k=0}^{n-1}\left(\int_{\substack{\left[-\tau-\left|\Lambda_{k+1}\right|,-\tau\right] \\
\cup\left[\tau, \tau+\left|\Gamma_{k+1}\right|\right]}}\left|f(t) w_{k+1}(t)\right| \mathrm{d} t\right) \frac{\left|\phi_{k}\left(t_{0}\right)\right|}{W_{k}} \\
& +\sup _{\substack{\|\vec{\Delta}\|_{p_{2}} \leq \delta \\
=}}\left|\sum_{k=0}^{n-1} \Delta_{k+1} \frac{\phi_{k}\left(t_{0}\right)}{W_{k}}\right| \\
& r(0,0)+\sup _{\substack{f \in E \\
\|\vec{\Gamma}\| p_{1} \leq \gamma \\
\|\bar{A}\|_{p_{1} \leq \gamma} \leq \gamma}} \sum_{k=0}^{n-1} A_{k}\left(f, \Gamma_{k+1}, \Lambda_{k+1}\right)\left|\phi_{k}\left(t_{0}\right)\right|+B \delta .
\end{aligned}
$$


If an $f$ belongs to $E$, then $|f(t)| \leq \sqrt{2 \Omega}$. Furthermore, the eigenrelations presented in Theorem 1 yield $\left|\phi_{k}(t)\right| \leq \sqrt{\frac{\Omega}{\pi \lambda_{k}}}$. Hence

$$
A_{k}\left(f, \Gamma_{k+1}, \Lambda_{k+1}\right) \leq \sqrt{\frac{2}{\pi \lambda_{k}}} \Omega\left(\left|\Gamma_{k+1}\right|+\left|\Lambda_{k+1}\right|\right) .
$$

This can be improved if $\gamma$ is small enough.

Lemma 5. If $f \in E, c \geq 2$ and

$$
\Omega \gamma<\frac{2}{\max _{i=0, \ldots, n-1} \beta_{i}},
$$

then

$$
A_{k}\left(f, \Gamma_{k+1}, \Lambda_{k+1}\right) \leq 2 \sqrt{\frac{\mathrm{d} \ln \lambda_{k}}{\mathrm{~d} c}} \Omega\left(\left|\Gamma_{k+1}\right|+\left|\Lambda_{k+1}\right|\right) .
$$

Proof. Since $|f(t)| \leq \sqrt{2 \Omega}$ and $\phi_{k}$ is odd for odd $k$ and even when $k$ is even, we obtain

$$
A_{k}\left(f, \Gamma_{k+1}, \Lambda_{k+1}\right) \leq \sqrt{2 \Omega} \int_{\tau}^{\tau+\left|\Lambda_{k+1}\right|}\left|\phi_{k}(t)\right| \mathrm{d} t+\int_{\tau}^{\tau+\left|\Gamma_{k+1}\right|}\left|\phi_{k}(t)\right| \mathrm{d} t .
$$

Expanding $\phi_{k}$ in the Taylor series and applying Corollary 1] we get

$$
\begin{aligned}
\int_{\tau}^{\tau+\left|\Gamma_{k+1}\right|}\left|\phi_{k}(t)\right| \mathrm{d} t & \leq \int_{\tau}^{\tau+\left|\Gamma_{k+1}\right|} \sum_{j=0}^{\infty} \frac{\left|\phi_{k}^{(j)}(\tau)\right|}{j !}(t-\tau)^{j} \mathrm{~d} t \\
& =\left|\phi_{k}(\tau)\right|\left|\Gamma_{k+1}\right|+\sum_{j=1}^{\infty} \frac{\left|\phi_{k}^{(j)}(\tau)\right|}{(j+1) !}\left|\Gamma_{k+1}\right|^{j+1} \\
& \leq\left|\phi_{k}(\tau)\right|\left|\Gamma_{k+1}\right|+\frac{2\left|\phi_{k}(\tau)\right|}{\Omega \beta_{k}} \sum_{j=1}^{\infty} \frac{\left(\frac{\Omega \beta_{k}}{2}\left|\Gamma_{k+1}\right|\right)^{j+1}}{j(j+1)} \\
& =\left|\phi_{k}(\tau)\right|\left|\Gamma_{k+1}\right|+\frac{2\left|\phi_{k}(\tau)\right|}{\Omega \beta_{k}}\left(x+\ln (1-x)^{1-x}\right),
\end{aligned}
$$

where $x=\frac{\Omega \beta_{k}}{2}\left|\Gamma_{k+1}\right|$. The last equation holds since $x<1$ is guaranteed by the assumptions. As $\ln (1-x)<0$ for $x \in[0,1)$, it follows that $x+(1-x) \ln (1-x) \leq x$. According to Lemma 1, we have $\left|\phi_{k}(\tau)\right|=\sqrt{\frac{\Omega}{2} \frac{\mathrm{d} \ln \lambda_{k}}{\mathrm{~d} c}}$. Consequently

$$
\int_{\tau}^{\tau+\left|\Gamma_{k+1}\right|}\left|\phi_{k}(t)\right| \mathrm{d} t \leq 2\left|\phi_{k}(\tau)\right|\left|\Gamma_{k+1}\right|=2 \sqrt{\frac{\Omega}{2} \frac{\mathrm{d} \ln \lambda_{k}}{\mathrm{~d} c}}\left|\Gamma_{k+1}\right| .
$$

The same argument applies to the integral $\int_{\tau}^{\tau+\left|\Lambda_{k+1}\right|}\left|\phi_{k}(t)\right| \mathrm{d} t$. The proof is complete.

We can now establish an upper bound on the radius of information.

Theorem 2. Assume that $c \geq 2$ and $\Omega \gamma<\frac{2}{\max _{i=0, \ldots, n-1} \beta_{i}}$. Then

$$
r(\gamma, \delta) \leq r(0,0)+A \Omega^{\frac{3}{2}} \gamma+B \delta,
$$


where

$$
\begin{aligned}
& A=\frac{4}{\sqrt{c}}\left\|\left[\sqrt{\frac{\mathrm{d} \ln \lambda_{0}}{\mathrm{~d} c}} v_{0}\left(\frac{t_{0}}{\tau}\right), \ldots, \sqrt{\frac{\mathrm{d} \ln \lambda_{n-1}}{\mathrm{~d} c}} v_{n-1}\left(\frac{t_{0}}{\tau}\right)\right]^{T}\right\|_{q_{1}}, \\
& B=\sqrt{\frac{\Omega}{c}}\left\|\left[\frac{1}{W_{0}} v_{0}\left(\frac{t_{0}}{\tau}\right), \ldots, \frac{1}{W_{n-1}} v_{n-1}\left(\frac{t_{0}}{\tau}\right)\right]^{T}\right\|_{q_{2}} .
\end{aligned}
$$

Proof. The theorem follows from Lemmas 4 and 5 and the definitions of $\phi_{k}$ and $c$.

One may ask whether the estimate in Theorem 2 is sharp. To answer this, we shall show a lower bound on the radius of information. The basic idea of getting it is to find two functions sharing the same information. Then we shall use them to estimate the diameter of information and finally the radius of information.

The following assumptions will be needed in the remainder of this section. They are a consequence of the proof technique, and all, except the first one, state some limitation on the size of $\gamma$. The goal of the last one is to guarantee that the functions $\phi_{k}, k=0, \ldots, n-1$, are nonnegative in the interval $[\tau-\gamma, \tau+\gamma]$.

A0. $\quad c \geq 2$,

A1. $\Omega \gamma \leq \frac{\pi}{12}$,

A2. $\quad \Omega \gamma \leq \frac{2\left\|\left[\lambda_{0} \beta_{0}, \ldots, \lambda_{n-1} \beta_{n-1}\right]^{T}\right\|_{p_{1}}}{\max _{i=0, \ldots, n-1} \beta_{i}}$,

A3. $\quad \Omega \gamma \leq \frac{1}{\max _{i=0, \ldots, n-1} \beta_{i}}$,

A4. $\quad \Omega \gamma$ such that $\forall_{k=0, \ldots, n-1} \forall_{t \in\left[1-\frac{2 \Omega \gamma}{c}, 1+\frac{2 \Omega \gamma}{c}\right]} v_{k}(t) \geq 0$.

Roughly speaking, the next lemma says that $\phi_{k}(\tau)$ is close to the mean value of the function $\phi_{k}$ on the interval $\left[\tau-\Gamma_{k+1}, \tau+\Gamma_{k+1}\right]$.

Lemma 6. If $c \geq 2, \Gamma_{k+1} \geq 0$ and $\Omega \beta_{k} \Gamma_{k+1}<1$, then

$$
\int_{\tau-\Gamma_{k+1}}^{\tau+\Gamma_{k+1}} \phi_{k}(t) \mathrm{d} t \geq \phi_{k}(\tau) \Gamma_{k+1}\left(2-\frac{1}{11}\left(\Omega \beta_{k} \Gamma_{k+1}\right)^{2}\right) \geq \frac{21}{11} \phi_{k}(\tau) \Gamma_{k+1} .
$$

Proof. The Taylor expansion of $\phi_{k}$ combined with Corollary 1 yields

$$
\begin{aligned}
& \left|\int_{\tau-\Gamma_{k+1}}^{\tau+\Gamma_{k+1}} \phi_{k}(t) \mathrm{d} t-2 \phi_{k}(\tau) \Gamma_{k+1}\right| \\
& =\left|\int_{\tau-\Gamma_{k+1}}^{\tau+\Gamma_{k+1}}\left(\sum_{j=0}^{\infty} \frac{\phi_{k}^{(j)}(\tau)}{j !}(t-\tau)^{j}\right) \mathrm{d} t-2 \phi_{k}(\tau) \Gamma_{k+1}\right| \\
& =\left|2 \sum_{j=1}^{\infty} \frac{\phi_{k}^{(2 j)}(\tau)}{(2 j+1) !} \Gamma_{k+1}^{2 j+1}\right| \\
& \leq \phi_{k}(\tau) \frac{2}{\Omega \beta_{k}} \sum_{j=1}^{\infty} \frac{2\left(\frac{\Omega \beta_{k}}{2} \Gamma_{k+1}\right)^{2 j+1}}{2 j(2 j+1)} .
\end{aligned}
$$


We set $x=\frac{\Omega \beta_{k}}{2} \Gamma_{k+1}$ and note that

$$
\sum_{j=1}^{\infty} \frac{2 x^{2 j+1}}{2 j(2 j+1)}=2 x+(1-x) \ln (1-x)-(1+x) \ln (1+x) \leq \frac{4}{11} x^{3}
$$

since $x \in\left[0, \frac{1}{2}\right)$. Consequently,

$$
\begin{aligned}
\left|\int_{\tau-\Gamma_{k+1}}^{\tau+\Gamma_{k+1}} \phi_{k}(t) \mathrm{d} t-2 \phi_{k}(\tau) \Gamma_{k+1}\right| & \leq \frac{\left(\Omega \beta_{k} \Gamma_{k+1}\right)^{2}}{11} \phi_{k}(\tau) \Gamma_{k+1} \\
& \leq \frac{1}{11} \phi_{k}(\tau) \Gamma_{k+1},
\end{aligned}
$$

and the lemma follows.

The task is now to find two functions sharing the same information and whose difference at some point $t_{0}$ is of order $\Omega^{\frac{3}{2}} \gamma$.

Lemma 7. Define

$$
\begin{aligned}
& \alpha=\frac{1}{2\left\|\left[\lambda_{0} \beta_{0}, \ldots, \lambda_{n-1} \beta_{n-1}\right]^{T}\right\|_{p_{1}}}, \\
& f_{1}(t)=\sqrt{2 \Omega} \operatorname{Sinc}(\Omega(t-\tau-\alpha \gamma)), \\
& f_{2}(t)=\sqrt{2 \Omega} \operatorname{Sinc}(\Omega(t-\tau+\alpha \gamma))
\end{aligned}
$$

and assume that $\delta=0$ and let conditions $A 0-A 4$ hold. Then the functions $f_{1}, f_{2}$ belong to $E$ and $\mathbb{N}\left(f_{1}\right) \cap \mathbb{N}\left(f_{2}\right) \neq \emptyset$.

Proof. Since

$$
\sqrt{2 \Omega} \operatorname{Sinc}(\Omega t)=\frac{1}{\sqrt{2 \Omega}} \int_{-\Omega}^{\Omega} e^{i t \omega} \mathrm{d} \omega,
$$

we get $f_{1}, f_{2} \in E$. In order to show that $\mathbb{N}\left(f_{1}\right) \cap \mathbb{N}\left(f_{2}\right) \neq \emptyset$, we need to find a vector $\vec{\Gamma}$ such that $\|\vec{\Gamma}\|_{p_{1}} \leq \gamma$ and

$$
\int_{-\tau}^{\tau-\Gamma_{k}} f_{1}(t) w_{k}(t) \mathrm{d} t=\int_{-\tau}^{\tau+\Gamma_{k}} f_{2}(t) w_{k}(t) \mathrm{d} t,
$$

for all $k=1, \ldots, n$. By the definition of $w_{k}$ and Theorem 1 we can rewrite the above equation as

$$
\begin{aligned}
\frac{\pi}{\Omega} \lambda_{k-1} & \left(\phi_{k-1}(\tau+\alpha \gamma)-\phi_{k-1}(\tau-\alpha \gamma)\right) \\
& =\int_{\tau}^{\tau+\Gamma_{k}} \operatorname{Sinc}(\Omega(t-\tau+\alpha \gamma)) \phi_{k-1}(t) \mathrm{d} t+\int_{\tau-\Gamma_{k}}^{\tau} \operatorname{Sinc}(\Omega(t-\tau-\alpha \gamma)) \phi_{k-1}(t) \mathrm{d} t
\end{aligned}
$$

where $k=1, \ldots, n$. According to $\mathrm{A} 1$ and $\mathrm{A} 4$ both integrants are nonnegative. Without loss of generality we assume that $\Gamma_{k} \geq 0$. Expanding $\phi_{k-1}$ in the Taylor 
series and applying Corollary 1 and property A2, we get

$$
\begin{array}{r}
\frac{\pi}{\Omega} \lambda_{k-1}\left(\phi_{k-1}(\tau+\alpha \gamma)-\phi_{k-1}(\tau-\alpha \gamma)\right) \\
\leq \frac{2 \pi}{\Omega} \lambda_{k-1} \phi_{k-1}(\tau) \sum_{j=0}^{\infty} \frac{\left(\frac{\alpha \beta_{k-1} \Omega \gamma}{2}\right)^{2 j+1}}{2 j+1} \\
\quad=\frac{2 \pi}{\Omega} \lambda_{k-1} \phi_{k-1}(\tau) \operatorname{arctanh} \frac{\alpha \beta_{k-1} \Omega \gamma}{2} .
\end{array}
$$

On the other hand, by A1, A3, and Lemma 6 it follows that

$$
\begin{aligned}
& \int_{\tau}^{\tau+\Gamma_{k}} \operatorname{Sinc}(\Omega(t-\tau+\alpha \gamma)) \phi_{k-1}(t) \mathrm{d} t+\int_{\tau-\Gamma_{k}}^{\tau} \operatorname{Sinc}(\Omega(t-\tau-\alpha \gamma)) \phi_{k-1}(t) \mathrm{d} t \\
& \quad \geq \frac{3}{\pi} \int_{-\tau-\Gamma_{k}}^{\tau+\Gamma_{k}} \phi_{k-1}(t) \mathrm{d} t \geq \frac{63}{11 \pi} \phi_{k-1}(\tau) \Gamma_{k} .
\end{aligned}
$$

We complete the proof by finding a vector $\vec{\Gamma}$ such that $\|\vec{\Gamma}\|_{p_{1}} \leq \gamma$ and

$$
\frac{2 \pi}{\Omega} \lambda_{k-1} \phi_{k-1}(\tau) \operatorname{arctanh} \frac{\alpha \beta_{k-1} \Omega \gamma}{2} \leq \frac{63}{11 \pi} \phi_{k-1}(\tau) \Gamma_{k} .
$$

To this end we set $\Gamma_{k}=2 \lambda_{k-1} \beta_{k-1} \alpha \gamma$. Then $\|\vec{\Gamma}\|_{p_{1}}=\gamma$ and the inequality above reduces to the inequality

$$
\operatorname{arctanh} \frac{\alpha \beta_{k-1} \Omega \gamma}{2} \leq \frac{126}{11 \pi^{2}} \frac{\alpha \beta_{k-1} \Omega \gamma}{2},
$$

which is valid since A2 implies $\frac{\alpha \beta_{k-1} \Omega \gamma}{2} \leq \frac{1}{2}$.

The following result is a counterpart of Theorem 2

Theorem 3. Under the assumptions A0-A4 we have

$$
r(\gamma, \delta) \geq \frac{1}{2}\left(r(0,0)+A \Omega^{\frac{3}{2}} \gamma+B \delta\right)+O\left(\gamma^{3}+\delta^{2}\right),
$$

where

$$
\begin{aligned}
A & =\frac{\sqrt{2}}{2} \frac{\max \left\{\left|\operatorname{Sinc}^{\prime}\left(\Omega\left(t_{0}-\tau\right)\right)\right|,\left|\operatorname{Sinc}^{\prime}\left(\Omega\left(t_{0}+\tau\right)\right)\right|\right\}}{\left\|\left[\lambda_{0} \beta_{0}, \ldots, \lambda_{n-1} \beta_{n-1}\right]^{T}\right\|_{p_{1}}}, \\
B & =\sqrt{\frac{\Omega}{c}}\left\|\left[\frac{1}{W_{0}} v_{0}\left(\frac{t_{0}}{\tau}\right), \ldots, \frac{1}{W_{n-1}} v_{n-1}\left(\frac{t_{0}}{\tau}\right)\right]^{T}\right\|_{q_{2}} .
\end{aligned}
$$

Proof. We define $f_{1}$ and $f_{2}$ as in Lemma 7 Since $\mathbb{N}\left(f_{1}\right) \cap \mathbb{N}\left(f_{2}\right) \neq \emptyset$, we have

$$
\begin{aligned}
d(\gamma, 0) & \geq\left|S\left(f_{1}\right)-S\left(f_{2}\right)\right| \\
& =\sqrt{2 \Omega}\left|\operatorname{Sinc}\left(\Omega\left(t_{0}-\tau-\alpha \gamma\right)\right)-\operatorname{Sinc}\left(\Omega\left(t_{0}-\tau+\alpha \gamma\right)\right)\right| \\
& =\frac{\sqrt{2}\left|\operatorname{Sinc}^{\prime}\left(\Omega\left(t_{0}-\tau\right)\right)\right|}{\left\|\left[\lambda_{0} \beta_{0}, \ldots, \lambda_{n-1} \beta_{n-1}\right]^{T}\right\|_{p_{1}}} \Omega^{\frac{3}{2}} \gamma+O\left(\gamma^{3}\right) .
\end{aligned}
$$

By considering the functions

$$
\begin{aligned}
& g_{1}(t)=\sqrt{2 \Omega} \operatorname{Sinc}(\Omega(t+\tau-\alpha \gamma)), \\
& g_{2}(t)=\sqrt{2 \Omega} \operatorname{Sinc}(\Omega(t+\tau+\alpha \gamma))
\end{aligned}
$$


and reasoning as in the proof of Lemma 7 we obtain that $\mathbb{N}\left(g_{1}\right) \cap \mathbb{N}\left(g_{2}\right) \neq \emptyset$ and consequently

$$
d(\gamma, 0) \geq \frac{\sqrt{2}\left|\operatorname{Sinc}^{\prime} \Omega\left(t_{0}+\tau\right)\right|}{\left\|\left[\lambda_{0} \beta_{0}, \ldots, \lambda_{n-1} \beta_{n-1}\right]^{T}\right\|_{p_{1}}} \Omega^{\frac{3}{2}} \gamma+O\left(\gamma^{3}\right) .
$$

Hence $d(\gamma, 0) \geq 2 A \Omega^{\frac{3}{2}} \gamma+O\left(\gamma^{3}\right)$. By [4] we also have $r(0, \delta)=r(0,0)+B \delta+O\left(\delta^{2}\right)$. We complete the proof by observing that

$$
r(\gamma, \delta) \geq \frac{1}{2} r(0, \delta)+\frac{1}{2} r(\gamma, 0) \geq \frac{1}{2} r(0, \delta)+\frac{1}{4} d(\gamma, 0) .
$$

We summarise Theorems 2 and 3 in the following final result.

Theorem 4. Let assumptions A0-A4 be satisfied. Then

$$
\frac{r(0,0)+A_{1} \Omega^{\frac{3}{2}} \gamma+B \delta}{2}+O\left(\gamma^{3}+\delta^{2}\right) \leq r(\gamma, \delta) \leq r(0,0)+A_{2} \Omega^{\frac{3}{2}} \gamma+B \delta,
$$

with quantities $A_{1}, A_{2}, B$ defined by the equations

$$
\begin{aligned}
A_{1} & =\frac{\sqrt{2}}{2} \frac{\max \left\{\left|\operatorname{Sinc}^{\prime}\left(\Omega\left(t_{0}-\tau\right)\right)\right|,\left|\operatorname{Sinc}^{\prime}\left(\Omega\left(t_{0}+\tau\right)\right)\right|\right\}}{\left\|\left[\lambda_{0} \beta_{0}, \ldots, \lambda_{n-1} \beta_{n-1}\right]^{T}\right\|_{p_{1}}}, \\
A_{2} & =\frac{4}{\sqrt{c}}\left\|\left[\sqrt{\frac{\mathrm{d} \ln \lambda_{0}}{\mathrm{~d} c}} v_{0}\left(\frac{t_{0}}{\tau}\right), \ldots, \sqrt{\frac{\mathrm{d} \ln \lambda_{n-1}}{\mathrm{~d} c}} v_{n-1}\left(\frac{t_{0}}{\tau}\right)\right]^{T}\right\|_{q_{1}}, \\
B & =\sqrt{\frac{\Omega}{c}}\left\|\left[\frac{1}{W_{0}} v_{0}\left(\frac{t_{0}}{\tau}\right), \ldots, \frac{1}{W_{n-1}} v_{n-1}\left(\frac{t_{0}}{\tau}\right)\right]^{T}\right\|_{q_{2}} .
\end{aligned}
$$

Remark. In order to make the radius of information sufficiently small, we may need to ensure that $A \Omega^{\frac{3}{2}} \gamma<1$ where $A \in\left[\min \left\{A_{1}, A_{2}\right\}, \max \left\{A_{1}, A_{2}\right\}\right]$. The quantities $A_{1}$ and $A_{2}$ depend only on $c$ and although they are not very close, the last theorem carries a negative message to practical applications. Usually relatively small values of $c$ are of interest. Taking $c \approx 3$ and $n$ not too big, but causing $r(0,0)$ to be small enough, we get $A_{1} \approx 0.1$. As we mentioned before, $\Omega$ can even exceed $2^{16}$ which leads to the inequality $\gamma<\frac{10}{2^{24}}$.

The measurement errors affect the radius of information $r(\gamma, \delta)$ depending on the way in which the functions $w_{1}, \ldots, w_{n}$ are scaled. Taking for example $W_{0}=$ $\cdots=W_{n-1}=\sqrt{\Omega}$, we get rid of the parameter $\Omega$ in the constant $B$.

\section{REFERENCES}

[1] D. Dąbrowska and M. A. Kowalski, Approximating band-and energy-limited signals in the presence of jitter, J. Complexity 14 (1998), 557-570. MR 2000d:65241

[2] C. Flammer, Spheroidal Wave Functions, Stanford University Press, Stanford, 1957. MR 19:689a

[3] W. H. J. Fuchs, On the eigenvalues of an integral equation arising in the theory of bandlimited signals, J. Math. Anal. Appl. 9 (1964), 317-330. MR 30:4128

[4] B. Z. Kacewicz and M. A. Kowalski, Approximating linear functionals on unitary spaces in the presence of bounded data errors with applications to signal recovery, J. Adaptive Control Signal Process. 9 (1995), 19-31. MR 95m:94001

[5] _ Recovering linear operators from inaccurate data, J. Complexity 11 (1995), 227-239. MR 96d:65104 
[6] _ "Recovering signals from inaccurate data" in Curves and Surfaces in Computer Vision and Graphics II (M. J. Silbermann and H. D. Tagare, Eds.), Proc. SPIE, Vol. 1610, Int. Soc. Opt. Eng., Bellingham, WA, (1992), pp. 68-74.

[7] M. A. Kowalski, Optimal complexity recovery of band- and energy-limited signals, J. Complexity 2 (1989), 239-254. MR 89i:94009

[8] , On approximation of band-limited signals, J. Complexity 5 (1989), 283-302. MR 90i:94006

[9] M. A. Kowalski, K. A. Sikorski, and F. Stenger, Selected topics in approximation and computation, Oxford University Press, New York, 1995. MR 97k:41001

[10] M. A. Kowalski and F. Stenger, Optimal complexity recovery of band- and energy-limited signals II, J. Complexity 5 (1989), pp. 45-49. MR 90c:41003

[11] H. J. Landau, The eigenvalue behavior of certain convolution equations, Trans. Amer. Math. Soc. 115 (1965), pp. 242-256. MR 33:7888

[12] _ Sampling, data transmission, and the Nyquist rate, Proc. IEEE 55 (1967), pp. 17011706.

[13] _ "An overview of time and frequency limiting" in Fourier Techniques and Applications (J. F. Price, Ed.), Plenum, New York, 1985.

[14] H. J. Landau and H. O. Pollak, Prolate spheroidal wave functions, Fourier analysis and uncertainty - II, Bell System Tech. J. 40 (1961), pp. 65-84. MR 25:4147

[15] _ Prolate spheroidal wave functions, fourier analysis and uncertainty - III. The dimension of the space of essentially time- and band-limited signals, Bell System Tech. J. 41 (1962), pp. 1295-1336. MR 26:5200

[16] A. A. Melkman, " $n$-Widths and optimal interpolation of time- and band-limited functions" in Optimal Estimation in Approximating Theory (C. A. Micchelli and T. J. Rivlin, Eds.), Plenum, New York, 1977. MR 57:956

[17],$n$-Widths and optimal interpolation of time- and band-limited functions II, SIAM J. Math. Anal. 16 (1985), pp. 803-813. MR 86h:41026

[18] C. A. Micchelli and T. J. Rivlin, "A survey of optimal recovery" in Optimal Estimation in Approximating Theory (C. A. Micchelli and T. J. Rivlin, Eds.), Plenum, New York, 1977. MR 56:3498

[19] L. Plaskota, Noisy Information and Computational Complexity, Cambridge Univ. Press, Cambridge, 1996. MR 99b:65189

[20] D. Slepian, Some asymptotic expansions for prolate spheroidal wave functions, J. of Mathematics and Physics 44 (1965), pp. 99-143. MR 31:3640

[21] — On bandwidth, Proc. IEEE 64 (1976), pp. 292-300. MR 57:2738

[22] D. Slepian and H. O. Pollak, Prolate spheroidal wave functions, Fourier analysis and uncertainty - I, Bell System Tech. J. 40 (1961), pp. 46-64. MR 25:4146

[23] J. F. Traub, G. W. Wasilkowski, and H. Woźniakowski, Information Based Complexity, Academic Press, New York, 1988. MR 90f:68085

[24] _ Information, Uncertainty, Complexity, Addison-Wesley, Reading, Mass., 1983. MR 85g:68031

[25] J. F. Traub and H. Woźniakowski, A General Theory of Optimal Algorithms, Academic Press, New York, 1980. MR 84m:68041

Faculty of Mathematics and Science, Cardinal Stefan Wyszyński University in WarSAW, ul. Dewajtis 5, 01-815 Warsaw, Poland

E-mail address: dabrowska@uksw.edu.pl 